\title{
MII RetCam assisted smartphone-based fundus imaging (MSFI)—A boon for paediatric retinal imaging
}

\author{
Rajwinder Kaur ${ }^{1} \cdot$ Harijot Singh ${ }^{1} \cdot$ Shallu Samria ${ }^{1} \cdot$ Nilesh Kumar $^{2} \cdot$ Nikulaa Parachuri $^{2} \cdot$ Rohini Sharma $^{3}$. \\ Francesco Bandello ${ }^{4}$ - Anat Loewenstein ${ }^{5}$ - Yannick Bilong ${ }^{6} \cdot$ M. Hafeez Faridi ${ }^{7}$. Ashish Sharma ${ }^{2}$
}

Received: 24 January 2020 / Revised: 7 February 2020 / Accepted: 11 February 2020

(c) The Royal College of Ophthalmologists 2020

Ophthalmologists have been fascinated with methods of viewing the retina, starting with the invention of the first ophthalmoscope by Hermann von Helmholtz in 1851 [1]. It has undergone innumerable modifications and reinventions and is still evolving. Fundus imaging of the human eye is an important diagnostic tool in ophthalmology.

Retinal disorders in children pose a special challenge to the retina specialist and so is fundus imaging. Patience and tact is needed in handling the children which allows avoiding unnecessary anaesthetic administration. To be able to perform important clinical examinations quickly can be a valuable asset in the arsenal of paediatric ophthalmologists. RetCam (Natus Medical System Inc., Pleasanton, CA, USA) is the established imaging device for ROP imaging [2]. It is cost-prohibitive and hence, has not been adopted widely in the developing countries such as India. Recent ophthalmic innovations have shown that smartphones are able to capture the fundus images with or without any

Ashish Sharma

drashish79@hotmail.com

1 Adesh Institute of Medical Sciences and Research, Bathinda, Punjab, India

2 Department of Vitreoretina, Lotus Eye Hospital and Institute, Coimbatore, Tamil Nadu, India

3 Department of Healthcare, The TIPS Global Institute, Coimbatore, Tamil Nadu, India

4 Department of Ophthalmology, University Vita-Salute, Scientific Institute San Raffaele, Milano, Italy

5 Division of Ophthalmology, Tel Aviv Sourasky Medical Center and Sackler Faculty of Medicine, Tel Aviv University, Tel Aviv, Israel

6 Departments of Eye, Faculty of Medicine and Biomedical Sciences, University of Yaounde I, Yaounde, Cameroon

7 College of Pharmacy, Chicago State University, Chicago, IL, USA attachment [3, 4]. MII RetCam is one such smartphonebased fundus imaging device which has been successfully utilised in ROP screening and tele-consultation programs, and also in the imaging of retinal lesions in adults [5-7]. This article highlights the use of MII RetCam as a tool for fundus imaging in the paediatric age group.

This is a single-centre; retrospective observational study for the utility of MII RetCam assisted smartphonebased fundus imaging (MSFI) as a part of the fundus imaging from July 2017 to November 2019. Fundus of babies who were admitted in the department of paediatrics in neonatal intensive care unit (NICU) and paediatric intensive care unit (PICU) were imaged. All premature babies were screened by trained ophthalmologist (RK) as per the request from the neonatologist (HS) according to the screening guidelines for ROP [8]. No neonates, infants or children were administered any form of anaesthesia for the evaluation and fundus imaging. This study also included children up to 15 years of age who were admitted in PICU and were also having retinal pathological manifestations. Such children were imaged for documentation, counseling and follow-up. The evaluation and imaging was performed by a single ophthalmologist. The pupil of all the patients was dilated prior to examination. Neonates were dilated using diluted tropicamide $(0.4 \%)$ with phenylephrine $(2.5 \%)$ while the older children were dilated using $0.8 \%$ tropicamide with $5 \%$ phenylephrine combination drops. Wire eye speculum was used for the ROP screening. One hand was used for holding MIIRetCam and other hand was used for rotation of the globe. One assistant's help was sought to stabilise the head of the child being imaged. (Fig. 1a)

Images were captured using a smartphone iPhone 5SE (Apple Inc., Cupertino, CA, USA) and 20D non-contact fundus lens (Volk Optical Inc., Mentor, OH, USA). 20D lens gives a field of view of 46 degrees. MII RetCam (Make in India RetCam), a smartphone-based fundus imaging device for capturing fundus images in premature babies and 

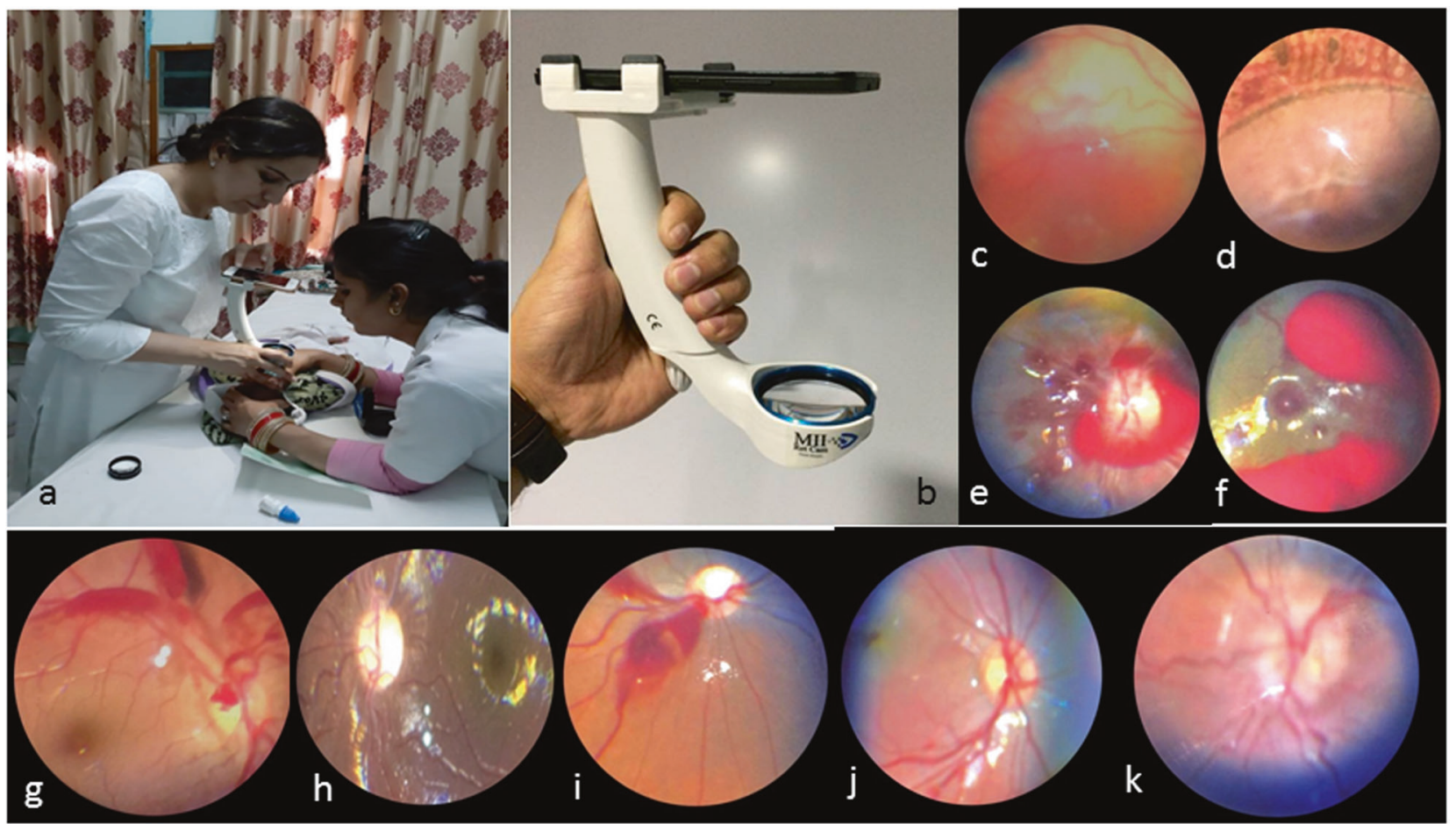

Fig. 1 MII RetCam assisted smartphone-based fundus imaging (MSFI) technique and paediatric retinal images in different diseases. a Showing the technique of MII RetCam assisted fundus imaging. b MII RetCam (Handheld). c Preterm with aggressive posterior retinopathy of prematurity (APROP). $\mathbf{d}$ Lasered lattice with peripheral shallow retinal detachment. e, f A 9-month-old baby diagnosed as battered baby syndrome showing preretinal haemorrhage and disc oedema. $\mathbf{g}$ A case of Terson's syndrome in a 13-year male child. $\mathbf{h}$ A 7-year-old child with lipemia retinalis. i A 12-year-old child with preretinal haemorrhages diagnosed as leukaemia. $\mathbf{j}$ A case of purtscher's retinopathy. $\mathbf{k}$ A child with papilloedema caused by benign intracranial hypertension secondary to nitrofurantoin. adults was innovated by Sharma et al. [9]. The device was designed to hold the lens and smartphone at a fixed working distance (Fig. 1b). The lens should be scratchfree to avoid artefacts and to have better autofocusing. Through a clear medium, the iPhone camera automatically focuses on the retina. The images were transferred using in-built app for storage and data transfer. Internet capabilities of the smartphone was used for group discussion and planning the management. The printouts were given to the paediatrician and parents of the child explaining the diagnosis and management.

A total of 50 children were initially imaged using MII RetCam. The inexpensive device was used for the flexibility and portability which allowed for the imaging to take place at the child's bed in all the cases. Overall, 37 (74\%) of the children were preterm babies who were imaged for ROP screening (Fig. 1c). All babies were awake during the examination.

There are many challenges in imaging the paediatric retina including the need for a wide-field image to capture the retina as peripherally as possible. This often requires a contact system to stabilise the globe. In a NICU setting with limited space, a separate bulky machine comes with logistical constraints. The ideal system will be a non-contact, portable, light-weight, preferably hand-held device which allows visualisation up to the ora serrata (Fig. 1d) with ease of image transfer.

Previously, fundus photos could only be taken on large, expensive cameras, which required patients in supine position to image the fundus, thus making ROP imaging very difficult. RetCam is the most widely used fundus imaging device for ROP imaging. Although it satisfies the criteria for an ideal system, but being so expensive, developing countries find it difficult to afford. The advent of smart phone fundus photography has been a boon for quick and low-cost documentation of the retina.

MSFI has been shown to be a potential imaging tool enabling objective documentation and monitoring of ROP in low resource setting [5-7]. In our study, MFSI was successful in documenting the fundus images, also helped the paediatrician in diagnosing and monitoring the status of seriously ill children up to 15 years of age. This article also highlights the teamwork of ophthalmologists and paediatricians in documentation of rare diseases such as battered baby syndrome (Fig. 1e, f), Tersons syndrome (Fig. 1g), lipemia retinalis (Fig. 1h), leukaemia (Fig. 1i), purtscher's 
retinopathy (Fig. 1j) and drug-induced benign intracranial hypertension (Fig. 1k).

Portability and financial affordability was a major factor making it a favourite choice as compared with the RetCam. Serial imaging helped the paediatrician in monitoring the course of disease over time by comparing with baseline photographs and planning appropriate management. Paediatrician found the images very helpful in counseling of the parents by ensuring better compliance with an added advantage of documentation for medicolegal reasons. In teaching institutes, fundus photo documentation can help residents in enriching their skills. Behera and Pradhan have also reported fundus photo documentation using MII RetCam in adult and paediatric cases evaluating its portability and efficacy [10].

MII RetCam is potential imaging tool for sharing and discussing the images for the betterment of the patient, a step towards giving the best care to the patient as well as a valuable tool in medicolegal situations in today's scenario.

\section{Compliance with ethical standards}

Conflict of interest The authors declare that they have no conflict of interest.

Publisher's note Springer Nature remains neutral with regard to jurisdictional claims in published maps and institutional affiliations.

\section{References}

1. Keeler SR. The ophthalmoscope in the lifetime of Hermann von Helmholtz. Arch Ophthalmol. 2002;120:194-201.

2. Chiang MF, Wang L, Busuioc M, Du YE, Chan P, Kane SA, et al. Telemedical retinopathy of premturity diagnosis: accuracy, reliability, and image quality. Arch Ophthalmol. 2007;125:1531-8.

3. Gunasekera CD, Thomas P. High-resolution direct ophthalmoscopy with an unmodified iPhone X. JAMA Ophthalmol. 2018;29:1-2.

4. Sharma A. Emerging simplified retinal imaging. Dev Ophthalmol. 2017;60:56-62.

5. Sharma A, Goyal A, Bilong Y, Shah P, Banker A, Kumar N. et al. Comparison of a smartphone-based photography method with indirect ophthalmoscopic assessment in referable retinopathy of prematurity (ROP): a smart ROP (S-ROP) model pilot study. Ophthalmol Retin. 2019;3:911-2.

6. Goyal A, Gopalakrishnan M, Anantharaman G, Chandrashekharan DP, Thachil T, Sharma A. Smartphone guided wide-field imaging for retinopathy of prematurity in neonatal intensive care unit-a Smart ROP (SROP) initiative. Indian J Ophthalmol. 2019;67:840-5.

7. Lekha T, Ramesh S, Sharma A, Abinaya G. MII RetCam assisted smartphone based fundus imaging for retinopathy of prematurity. Indian J Ophthalmol. 2019;67:834-9.

8. Jalali S, Anand R, Kumar H, Dogra MR, Azad R, Gopal L. Programme planning and screening strategy in retinopathy of prematurity. Indian J Ophthalmol. 2003;51:89-99.

9. Sharma A, Subramaniam SD, Ramachandran KI, Lakshmikanthan C, Krishna S, Sundaramuurthy SK. Smartphone-based fundus camera device (MIIRetCam) and techinique with ability to image peripheral retina. Eur J Ophthalmol. 2016;26:142-4.

10. Behera S, Pradhan A. MII Retcam: a boon towards retinal disease and imaging and documentation. J Clin Exp Ophthalmol. 2017;8:1-3. 